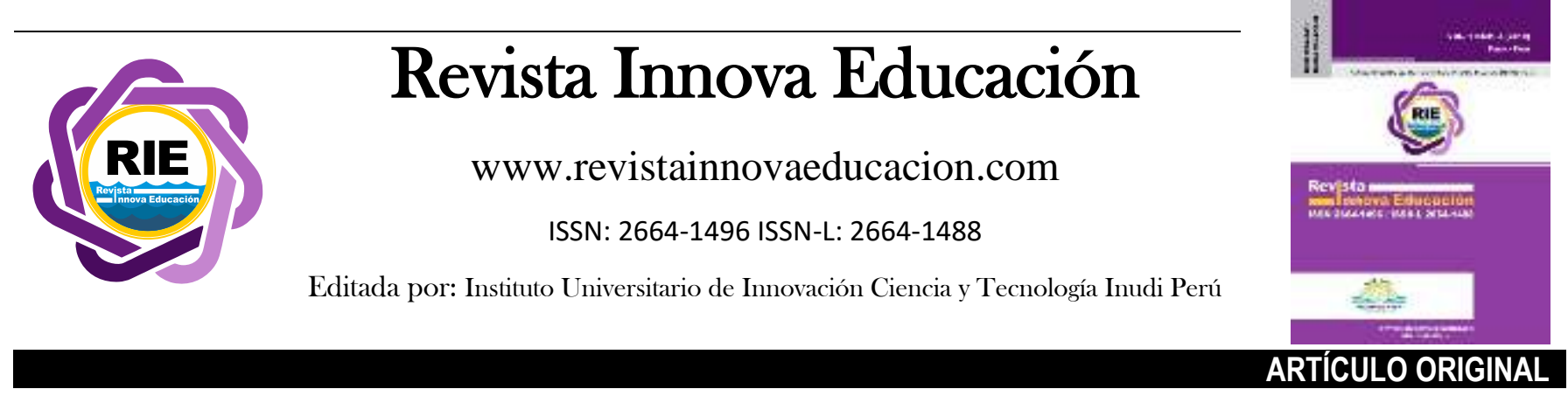

\title{
El papel de las creencias de los maestros en la participación familiar
}

\author{
The Role of Teachers' Beliefs in Family Engagement
}

O papel das crenças dos professores no envolvimento da família

\author{
Mauricio Rodríguez-Herrera ${ }^{1}$ \\ Universidad Autónoma de Tlaxcala, Tlaxcala - Tlaxcala, México \\ (D) https://orcid.org/0000-0002-2865-3172 \\ mrodriguez@uniquindio.edu.co (correspondencia)
}

\author{
DOI (Genérico) \\ DOI (Documento en español) \\ DOI (Document in English)
}

: https://doi.org/10.35622/j.rie.2021.01.008
: https://doi.org/10.35622/j.rie.2021.01.008.es
: https://doi.org/10.35622/j.rie.2021.01.008.en

Recibido 03/10/2020/ Aceptado 30/12/2020 Publicado 05/02/2021

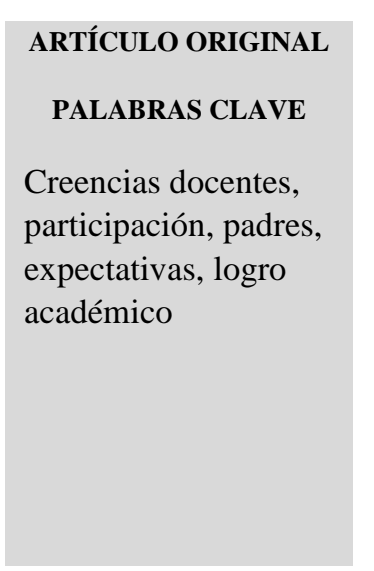

\section{KEYWORDS}

Teacher beliefs, participation, parents, expectations, academic achievement
RESUMEN. La escuela como institución no deja de ser una organización conformada por individuos, quienes pueden efectivamente alterar la realidad al interior de la estructura escolarizante. Este trabajo consistió en una exploración del papel de las creencias de los maestros en la participación familiar. Para ello se definieron las creencias docentes, logro académico, expectativas y participación familiar como conceptos fundamentales a la hora de comprender el papel explorado. El objetivo de este trabajo, evaluar cómo inciden las creencias de los docentes en la participación de los padres, se abordó desde una revisión de las principales investigaciones que al respecto se han generado sobre este capo del conocimiento educativo. Como eje central se profundiza efectivamente sobre las creencias y dentro de las principales conclusiones se resalta el que el fomento de la participación de los padres por parte de los maestros depende directamente de los imaginarios, la cultura y creencias que este grupo tiene sobre la incidencia positiva o negativa que tiene la participación de los padres.

\begin{abstract}
The school as an institution continues to be an organization made up of individuals, who can effectively alter reality within the school structure. This work consists of an exploration of the role of teachers' beliefs in family participation. For this, teaching beliefs, academic performance, expectations, and family participation were defined as fundamental concepts when it came to understanding the explored role. The objective of this work, to evaluate how teachers' beliefs affect parental participation, was approached from a review of the main investigations that have been generated regarding the field of educational knowledge. As the central axis, beliefs are effectively deepened and among the main conclusions, it is highlighted that the promotion of parental participation by teachers depends directly on the imaginary, culture, and beliefs that this group has on the positive impact or rejection of parental participation.
\end{abstract}

\footnotetext{
${ }^{1}$ Doctorando en educación en la Universidad Autónoma de Tlaxcala, México.
} 


\begin{tabular}{ll}
\hline PALAVRAS-CHAVE & $\begin{array}{l}\text { RESUMO. A escola como instituição continua a ser uma organização formada por } \\
\text { indivíduos, que podem efetivamente alterar a realidade dentro da estrutura escolar. Este }\end{array}$ \\
Crenças do professor, & $\begin{array}{l}\text { trabalho consistiu em uma exploração do papel das crenças dos professores na } \\
\text { participação, pais, }\end{array}$ \\
expectativas, & $\begin{array}{l}\text { participação familiar. Para eles, o ensino de crenças, desempenho acadêmico, } \\
\text { desempenho }\end{array}$ \\
acadêmico & a compreensão do papel explorado. O objetivo deste trabalho, avaliar como as crenças \\
& dos professores afetam a participação dos pais, foi abordado a partir de uma revisão \\
& das principais investigações que têm sido geradas a esse respeito neste campo do \\
& conhecimento educacional. Como eixo central, as crenças são efetivamente \\
& aprofundadas e entre as principais conclusões, destaca-se que a promoção da \\
& participação parental pelos professores depende diretamente do imaginário, da cultura \\
& e das crenças que esse grupo tem no impacto positivo ou na recusa em ter envolvimento \\
parental.
\end{tabular}

\section{INTRODUCCIÓN}

Sobre la educación recae la carga de considerarse el principal motor de escala social, sin embargo, esto no ha sido cierto para América Latina debido a que el aumento paulatino de la matrícula escolar no se ha traducido necesariamente en un desarrollo económico proporcional. En Latinoamérica, los estudios revelan que aún es muy alta la probabilidad de que quien nazca en un hogar pobre, muera pobre (Cárdenas, 2013).

Esta desafortunada tendencia ha sido interpretada por los investigadores como consecuencia de la calidad de la educación (Arreola, Carlos \& Valdés, 2013), por lo que encontrar elementos que contribuyen a una mejor educación tiene importancia estratégica para los intereses de desarrollo de las sociedades.

Frente a esto, las instituciones empiezan a comprender la necesidad de incluir a la sociedad en los diferentes asuntos institucionales, o, dicho de otra forma, el espacio escolar es abierto paulatinamente a la familia (Blanco \& Umayahara, 2004), así se requiere que los diferentes actores, estudiantes, padres de familia, docentes y la comunidad; sean más activos.

Según Freeman (2002), esto incluye la necesidad de tener en cuenta tanto el entorno principal en el que se desarrollan los estudiantes, es decir, donde inicialmente se relacionan con otros, como en la escuela y el hogar, y los entornos socioculturales donde se llevan a cabo la interacciones (la escuela, en el caso de las relaciones entre profesores, estudiantes y padres de familia). Esto con el fin de mejorar el aprendizaje y apoyar el desarrollo del estudiante.

Cabe señalar que la promoción de la asociación entre familia y escuela no tiene orígenes exclusivamente técnicos, los trabajos de Blanco y Umayahara (2004) y Corral, Frías, González y Miranda (1998), han asociado la capacidad de esfuerzo de los hijos con el afecto de los padres y la transmisión de valores y principios evidenciados a través de su involucramiento con la escuela. Al respecto, Miranda (1995) encuentra que la interacción entre la familia-escuela tiene importantes efectos en el desarrollo de la niñez.

Por lo anterior, comprender el rol de la familia en el desempeño escolar y cómo esta interacción fortalece la adaptación al contexto escolar del alumno, y a su vez influencia en los puntajes de los exámenes de conocimiento; es fundamental (Valdés, 2007). Esto es coherente con 
una investigación realizada por Felipe Martínez, quien fuera director del Instituto Nacional para la Evaluación de la Educación de México (INEE), sugiere que es el factor más influyente en el desempeño escolar son las prácticas familiares, incluso por encima de los aspectos demográficos, económicos y comunitarios que rodean al estudiante (Martínez, 2001).

En los últimos años han proliferado estudios internacionales que pretenden indagar sobre el impacto positivo que tiene la participación de las familias en la educación de sus hijos respecto al logro académico en aras de mejorar la calidad de la educación preescolar, primaria y secundaria al promover precisamente el involucramiento de los padres en la educación de sus hijos (Boonk, Gijselaersa, Ritzen \& Saskia, 2018; Bronfenbrenner \& Morris, 2016; Carlos, Urías, Valdéz \& Wendlandt, 2016; Arreola, Carlos \& Valdés, 2013; Hornby \& Lafaele, 2011; Green, Hoover, Sandler \& Walker, 2007; Bronfenbrenner \& Morris, 2006; Bertrand \& Deslandes, 2005; Hoover, Jones, Reed \& Walker, 2002; Torrico et al., 2002; Keyes, 2000; Corral, Frías, González \& Miranda, 1998; Hoover, Jones, Reed \& Walker, 2000; Hoover \& Sandler, 1997; Epstein, 1986; Bronfenbrenner, 1979). En este sentido y sobre la participación escolar es muy relevante el rol de los maestros, cuya contribución es estratégica para que puedan florecer las ventajas de la intervención de la familia.

Este trabajo explora en primer lugar, la relación familia-escuela en función de las implicaciones que esto tiene para el aprendizaje de las niñas y niños, para ello, profundiza en el concepto de participación de los maestros, el rol de los padres y principalmente la influencia de las creencias de estos actores sobre los resultados de aprendizaje; y, en segundo lugar, los principales modelos que sobre la relación familia-escuela se han extendido.

Este artículo genera algunas reflexiones acerca de la importancia de la participación de la familia en la escuela, pues, aunque sean dos unidades básicas en el proceso educativo, pareciera que están fragmentadas, ya que los maestros pueden no saber cómo estimular y mantener los esfuerzos de participación. En consecuencia, los padres cuya participación no es invitada pueden apreciar exclusión o poca consideración por su participación, lo que refleja la falta de estrategias efectivas que logren formar y fortalecer el vínculo familia-escuela.

Este vínculo maestro-padres, en función de la mejora en la participación de los padres puede ser contundente a la hora de mejorar los programas escolares, el clima escolar, el sentido de comunidad, el sentido de eficacia parental, la crianza positiva, mejorar el comportamiento del niño, adaptación escolar, habilidades académicas y competencia escolar.

\section{MÉTODO}

Este trabajo corresponde al método inductivo de tipo descriptivo y de diseño de análisis documental. Las informaciones se obtuvieron de diferentes bases de datos como: Scopus, Web of Sicence, ScienDirect, DOAJ, Ebsco y Google Académico. 
Tabla 1. Autores tratados según eje de estudio.

\begin{tabular}{|c|c|c|}
\hline $\begin{array}{c}\text { Universo de } \\
\text { investigación }\end{array}$ & $\begin{array}{l}\text { Ejes de } \\
\text { estudio }\end{array}$ & Principales teóricos \\
\hline \multirow{4}{*}{$\begin{array}{l}\text { El papel de las creencias } \\
\text { de los maestros en la } \\
\text { participación familiar }\end{array}$} & $\begin{array}{l}\text { Creencias } \\
\text { docentes }\end{array}$ & $\begin{array}{l}\text { - } \text { Ali, Alizadeh, y Tehranian (2018) } \\
\text { - } \quad \text { Bayas, Molina, Ordóñez, y Rodríguez (2018) } \\
\text { - Amaya, Delgado, Gnecco, León, y Valencia } \\
\text { (2014) } \\
\text { - } \quad \text { Fuentes, Guzmán, Quevedo, y Villablanca (2013) }\end{array}$ \\
\hline & $\begin{array}{l}\text { Logro } \\
\text { académico }\end{array}$ & $\begin{array}{ll}\text { - } & \text { Bazán, Hernández, Hernández, y Ochoa (2020) } \\
\text { - } & \text { Duckworth, Eskreis, Galla, Gross, y Taxer (2019) } \\
\text { - } & \text { Luna y Velázquez (2019) } \\
\text { - } & \text { Astorquiza (2018) } \\
\text { - } & \text { Meenu (2016) } \\
\text { - } & \text { Ramírez, Velasco, y Vera (2015) } \\
\end{array}$ \\
\hline & Expectativas & $\begin{array}{ll}\text { - } & \text { Anzaldo y Benavides (2020) } \\
\text { - } & \text { Becker y Wessling (2020) } \\
\text { - } & \text { Bendixsen y Danielsen (2020) } \\
\text { - } & \text { Daza y Garza (2020) } \\
\text { - } & \text { Danna, Londoño, y Stellian (2020) } \\
\text { - } & \text { Juang, Noack, Schachner, Vietze, y Vijver (2020) } \\
\text { - } & \text { Anzoátegui y Galvis, 2019) } \\
\end{array}$ \\
\hline & $\begin{array}{l}\text { El papel de } \\
\text { las creencias } \\
\text { de los } \\
\text { maestros en } \\
\text { la } \\
\text { participación } \\
\text { familiar }\end{array}$ & $\begin{array}{ll}\text { - } & \text { Coninck, Dotger, Vanderlinde y Walker (2020) } \\
\text { - } & \text { Eva y Mei (2019) } \\
\text { - } & \text { Guo y Kilderry (2018) } \\
\text { - } & \text { Martijn, Mutton, Thompson y Vanderlinde (2018) } \\
\text { - } & \text { Aguirre et al. (2013) } \\
\text { - } & \text { Derin, Noftle, Quintanar y Warren (2011) } \\
\text { - } & \text { Bakker, Brus y Denessen (2007) } \\
\text { - } & \text { Hoover, Jones, Reed y Walker (2002) } \\
\end{array}$ \\
\hline
\end{tabular}

Fuente: Elaboración propia

\section{RESULTADOS Y DISCUSIONES}

\subsection{Creencias docentes}

Las creencias de los docentes tienen directa influencia en sus percepciones y juicios y por lo tanto en sus acciones (Bayas, Molina, Ordóñez \& Rodríguez, 2018) si bien estas tienen relación con la construcción subjetiva de las expectativas, las creencias son "conjuntos de ideas integrados y consistentes, que se generan a partir de las experiencias cotidianas, son versiones personales de la realidad y cuentan con un nivel de organización interna que permiten una estructura, sistematicidad y una interpretación de los hechos" (Amaya, Delgado, Gnecco, León \& Valencia, 2014, pág. 50). Esta interpretación que se manifiesta en el ambiente influye tanto en las tomas de decisiones como en las prácticas docentes llevadas a cabo (Fuentes, Guzmán, Quevedo \& Villablanca, 2013), así las creencias, que pueden estar influenciadas por factores colectivos y ambientales, juegan un papel decisivo e innegable en el logro de objetivos y misiones de educación 
en términos cuantitativos y cualitativos ya que determinan sus métodos de enseñanza (Ali, Alizadeh \& Tehranian, 2018).

\subsection{Logro académico}

El logro académico ha sido relacionado tradicionalmente con la calidad educativa, siendo este una representación positiva o un nivel alto en las notas escolares (Ramírez, Velasco \& Vera, 2015; Astorquiza, 2018; Meenu, 2016; Duckworth, Eskreis, Galla, Gross \& Taxer, 2019), y ha sido principalmente relacionado con las disciplinas de matemáticas y español (Bazán, Hernández, Hernández \& Ochoa, 2020; Luna \& Velázquez, 2019).

Para este trabajo se entenderá como logro académico aquel que supone resultados favorables con respecto a los objetivos generales propuestos dentro del contexto escolar.

\subsection{Expectativas}

Las expectativas son ampliamente estudiadas en diversas disciplinas de la ciencia, desde la estadística hasta la educación. Este concepto está relacionado con la conducta de anticipar el valor que podría tomar algo en el futuro (Danna, Londoño \& Stellian, 2020), más propiamente con el valor que se espera que tome una variable (Anzaldo \& Benavides, 2020), depositando entonces un grado de confianza sobre un resultado a futuro (Anzoátegui \& Galvis, 2019).

Esto implica que las expectativas son una predisposición y constructo subjetivo sobre algo o alguien (Daza \& Garza, 2020) que afecta la percepción en el presente, en este caso, de un sujeto sobre otro (Juang, Noack, Schachner, Vietze \& Vijver, 2020). El problema radica en que las expectativas son prejuicios o pronósticos basados en múltiples variables como la cultura o los imaginarios (Becker \& Wessling, 2020; Bendixsen \& Danielsen, 2020) y afectan la relación o el trato de una persona que determine una postura frente a otra.

Las expectativas o juicios preconcebidos pueden ser positivos o negativos, pero siempre afectan la relación en el presente basado en la delimitación que un sujeto hace sobre el futuro de otro.

\section{a. El papel de las creencias de los maestros en la participación familiar}

Por su parte, Martijn, Mutton, Thompson y Vanderlinde (2018) subrayan que, a pesar de la evidencia teórica, los maestros no han dado la importancia que debe tener la familia en el proceso educativo dentro de la escuela. El poco valor que se le da a la opinión y participación de los padres dentro de la escuela puede estar afectando el potencial de aprendizaje de las niñas y niños y los maestros parecen no estar preparados para incorporar a la familia activamente e incluso, en ocasiones muestran resistencia a esto. Una de las causas de esta conducta puede ser que los maestros se sienten sobrecargados de trabajo y responsabilidad y ven la incorporación de la familia como una carga adicional.

Esto último es fundamental para entender por qué los cambios dentro del aula de clase no se dan de forma más acelerada a pesar de las múltiples investigaciones alrededor de la mejora en la calidad educativa. En definitiva, el actor principal que debe ejecutar las estrategias se siente 
sobrecargado. A esto se suma la necesidad de estar en disposición de aprender permanentemente para familiarizarse con los últimos cambios, lo cual sigue exigiendo al maestro más y más tareas. Además, el área de la microadministración educativa no tiene dentro de sus prioridades mejorar la estructura pedagógica.

Esto, según los planteamientos de Martijn, et al. (2018), implican que los desarrollos avanzados deben ir tanto al aula de clase como a las escuelas de preparación docente, para formar a los futuros maestros en dichas prácticas. Evidentemente el grado de exigencia es muy alto siempre que se requiera mejorar la educación dada la complejidad de factores socioeconómicos y culturales que intervienen. Martijn, et al. (2018) hace un barrido de las investigaciones al respecto en Estados Unidos, Australia, Inglaterra, Suiza, España, Finlandia, Noruega, Bélgica y los Países Bajos; y encuentra que sus hallazgos son comunes a todos los países.

Derin, Noftle, Quintanar y Warren (2011) también exploran esta necesidad de incluir este tema en el currículo de los futuros maestros y hacen un estudio en el que se incorpora este contenido a nivel posgradual para evidenciar el impacto que tiene este tipo de formación en las acciones docentes. Este estudio se llevó a cabo con una población de 157 docentes en dos universidades del sur de California y concluye que cuando los maestros valoran y aprecian las contribuciones de las familias y la comunidad, se pueden construir relaciones auténticas, las cuales resultan en mejores oportunidades educativas para los niños.

A estas mismas conclusiones llegaron Hoover, Jones, Reed y Walker (2002), quienes hicieron un estudio al sur de los Estados Unidos sobre un grupo experimental de 30 maestros y un grupo control de 22 maestros en 2 escuelas diferentes. Logrando identificar que es necesario que los docentes reciban preparación en estos temas durante su vida universitaria.

Estos referentes en el tema han decidido entender las diferencias entre las expectativas de los docentes y los padres como un dilema que implica que: "los maestros pueden no saber cómo invitar o mantener los esfuerzos de participación; y los padres que no son invitados a participar pueden percibir exclusión intencional o poca consideración por su participación" (Hoover, Jones, Reed \& Walker, 2002, pág. 844). A lo que se puede agregar, los maestros pueden no encontrar valor en la participación de los padres y los padres responden con menor participación debido a la ausencia de una invitación a participar, por lo cual se hace necesario formar a los docentes sobre la importancia de los padres en la educación de las niñas y niños.

En línea con esto, Eva y Mei (2019), las cuales entienden las asociaciones entre el hogar y la escuela se refieren a la colaboración entre la escuela y la familia para maximizar los resultados de aprendizaje de los estudiantes y abordan la relación familia-escuela en China. Para estos autores, el impacto positivo de la familia en la escuela no está en entredicho y por lo tanto se centran en los factores que han obstaculizado un mayor acercamiento entre estas partes, puesto que, los padres chinos son conocidos por su entusiasmo y dedicación a la educación de los niños como resultado de su fuerte creencia tradicional de que la educación es la clave del éxito, pero esta dedicación es más presente en el hogar y no tanto en la escuela.

Eva y Mei (2019), encuentran que ambos sujetos, escuela y familia, tienen una visión diferente de la buena educación y esto hace que no se integren completamente, a pesar de que 
mejora: programas escolares, clima escolar, moral escolar, sentido de comunidad, sentido de eficacia de los padres de familia, crianza positiva, comportamiento del niño, adaptación escolar, habilidades académicas y competencia escolar. En su investigación, las autoras encuentran que se puede enfatizar que la participación de los padres en las escuelas es una forma de capital social que contribuye a la comunidad escolar en general.

En este sentido, el alcance de Eva y Mei (2019) enfatiza en que las escuelas deben tener políticas claras y bien establecidas para colaborar con los padres, y fomentar la autopercepción de buen desempeño, ya que, siguiendo a Bandura, los autores encuentran que: "los padres que confían en su capacidad de promover el rendimiento académico de sus hijos tienen más probabilidades de tener niveles más altos de asociación en el hogar que los que no lo hacen" (Eva \& Mei, 2019, pág. 11). Para corroborar esta tesis, aplican como instrumento metodológico, la entrevista, sobre 3 grupos de ingresos diferentes en el cual participan 6 directivas, 9 maestras y 12 madres en Hong Kong, del cual extraen que las familias más involucradas en la escuela tienen mayor interés por reproducir y reforzar lo enseñado dentro de la escuela en su hogar y se sienten más empáticos con los docentes. En su estudio, las barreras también fueron analizadas y entre ellas se encuentran: la falta de tiempo de los padres debido al trabajo, tener hijos menores que requieren mayor atención, las tareas domésticas. Esto lo han solucionado con la divulgación de un calendario de actividades escolares anticipado. Pero no sólo es esto, en su investigación Eva y Mei (2019) encuentran que los maestros están acostumbrados a dar instrucciones que se sigan al pie de la letra y cuando los padres no lo hacen por estar pendientes de sus hijos, les exaspera. Además, los maestros opinan en general que la presencia de los padres en la escuela tiene efectos negativos ya que los niños actúan diferentes cuando les tienen cerca.

También en China, Guo y Kilderry (2018) profundizan sobre el papel de los maestros en la relación familia-escuela. Sin embargo, en el proceso, estas autores presentan las diferencias culturales como hechos trascendentales sobre los cuales estudiar la relación familia-escuela, pues las discrepancias entre las diferentes regiones y países da cuenta de un entendimiento no extendido de lo que se puede comprender como afecto, cuidado, atención y diversas palabras que se usan en el lenguaje académico formal para revelar el rol familiar, pero hay que clarificar qué significa cuidado para cada sujeto ya que de no interpretarse correctamente, la señal puede ser equivocada y la participación familiar se desdibuja en un esquema complejo y descontextualizado. En el caso particular China, la relación padres-hijos está mediada en su mayoría por la tradición milenaria del confusionismo y es bajo este marco por donde se debe dialogar con los padres para que mejoren sus prácticas familiares en relación con la participación de la educación de sus hijos.

Respecto a los maestros chinos, al igual que Eva y Mei (2019), Guo y Kilderry (2018) coinciden en que los maestros son vistos como figuras expertas y sus prácticas no sólo no son cuestionadas, sino que son respetadas hasta el punto de entender como terreno exclusivo del docente la educación de las niñas y niños, por lo cual, el gobierno chino ha tenido que intervenir con una política educativa que promueve la cooperación entre ambos actores. Esta política ha tenido un éxito de lento crecimiento gracias a la occidentalización paulatina del gigante asiático.

Coninck, Dotger, Vanderlinde y Walker (2020) tienen otra apreciación respecto a la brecha maestro-familia y esta se centra en el aspecto de la comunicación. Para estos autores, el problema 
radica en que los docentes no cuentan con las suficientes habilidades comunicativas para transmitir a las familias la importancia de su rol. La estrategia comunicativa predominante que se revisa es la de las conferencias, ya que se considera que estas son clave para establecer asociaciones eficaces entre familia y escuela, ya que ofrecen la oportunidad de intercambiar información sobre el alumno y de alinear los procesos educativos en la escuela y en el hogar. Idealmente, las conferencias familia-maestro implican un diálogo bidireccional, toma de decisiones y la identificación de acciones compartidas que los maestros, las familias y los estudiantes pueden emprender para avanzar en el éxito escolar de los estudiantes. No obstante, para que estos espacios sean aprovechados, las familias deben tener el mismo peso en la participación y la agenda de los maestros, es decir, no es una clase de los maestros a los padres, sino una reunión de iguales en donde el centro del diálogo es la educación de las niñas y niños.

Coninck, et al. (2020) tienen una postura respecto a la eficacia de los programas de aprendizaje el cual involucran a la familia-escuela, muy cercana a las creencias. Si bien, se ha investigado que son las creencias las que influyen en la forma en que la familia acompaña el aprendizaje de las niñas y niños, Coninck, et al. (2020) encuentran que la creencia de los maestros sobre sí mismo también influye. En este caso, si el maestro tiene un juicio positivo acerca de sus habilidades para promover el aprendizaje de los estudiantes, su nivel de involucramiento y, por ende, de sus resultados será mayor, en materia de involucrar activamente a la familia y promover estrategias eficaces de aprendizaje. Es decir, las creencias de los maestros en materia de su calidad profesional afectan en los maestros proporcionalmente su compromiso laboral, satisfacción laboral y bienestar. Es decir, las creencias de autoeficacia representan una variable afectiva-motivacional importante la cual sustenta un desempeño docente exitoso.

Aguirre et al. (2013) se adentra un poco más respecto a cómo influyen las creencias de los maestros en la relación familia-escuela y encuentra que, si los docentes tienen percepciones negativas acerca de nivel de aprendizaje o el valor de la cultura de etnias minoritarias o sesgos de género o si tienen algún prejuicio, estos van a limitar su propio proceso de enseñanza ya sea por subvaloración de una población minoritaria o sobrevaloración de una población específica. Estas creencias también pueden tener un sesgo en la medida en que los maestros pueden considerar que su opinión es objetiva, mientras que la de los demás actores, estudiantes y padres, es subjetiva. La cultura en esta investigación también es transversal, y es que si los mismos padres, ven a la escuela como única autoridad en materia de educación para sus hijos, su nivel de participación se restringe a seguir las indicaciones hechas por la escuela y no a participar en un proceso cooperativo.

Sobre esta influencia de las creencias, Bakker, Brus y Denessen (2007) estudian el impacto que tienen la percepción de los maestros sobre la relación familia-escuela. Estos autores inician mencionando que se tiende a encontrar empíricamente que existe un impacto positivo del capital cultural y social en la educación de las niñas y niños, y, en consecuencia, como las esferas con bajos índices socioeconómicos tienen menor rendimiento escolar. No obstante, se ha encontrado que tales diferencias se reducen a la efectiva participación de los padres en la educación de sus hijas e hijas y no exclusivamente al entorno socioeconómico. El trabajo de Bakker, et al. (2007) realizado sobre una muestra de 60 maestros y 218 padres en escuelas de Holanda, apunta a que los maestros consideran como deseable las formas de participación de la clase media y, por lo tanto, 
no visibilizan diversas formas de participación de las familias en condiciones de vulnerabilidad económica. Por lo que su comportamiento responde más a la presencia de profecías autocumplidas.

La investigación de Aguirre et al. (2013) también contribuye a reconocer formas más específicas de participación de la familia a través de relacionamiento de las disciplinas con el entorno, esto implica, el aprendizaje de matemáticas a través de la cocina, o de historia a través de paseos urbanos o de geografía por medio de viajes. Es decir, involucrar el aprendizaje conscientemente en la cotidianidad. En esto hay que tener precaución de malinterpretarlo, ya que la misma investigación sugiere que los maestros consideran como acertadas las prácticas académicas que se relacionan más con pertenecer a la clase media que con el aprendizaje en general, por ejemplo, tener cierto tipo de juguetes o tener cierta vida social o tipo de cultura o estar rodeado de cierto tipo de profesionales. La realidad es que en la práctica el aprendizaje sucede en los seres humanos (seres vivos en general) y no específicamente en una clase social determinada.

\section{RESULTADOS}

De la tradición de Bandura y respecto a entender al hombre como un sujeto social, algunos investigadores han encontrado que más que un proceso individual o que sucede al interior de las familias, la participación de los padres se reafirma en comunidad, una comunidad que es integrada tanto por otros padres como por los maestros y en general, por todos los actores educativos. Al actuar dentro de una comunidad los padres no sólo están favoreciendo el aprendizaje de sus hijos, sino de los estudiantes en general. Esto puede tener origen en que los estudiantes no son entes ajenos, sino que construyen lazos de amistad que transcienden incluso la familia. Salidas de campo, trabajos en grupo, reuniones familiares y visitas frecuentes a diferentes entornos familiares pueden reducir el efecto que pueda tener en un niño una familia que tenga baja participación de los padres o incluso incrementar los efectos positivos que tienen las familias que integran padres que participan activamente. En este sentido, lo que sucede es que se conforma una red que se fortalece con la participación de cada actor relevante dentro de la comunidad educativa (Holloway, Park \& Stone, 2017; Derin, Noftle, Quintanar \& Warren, 2011).

No obstante, para que esta red funcione debe existir canales funcionales de comunicación que permitan crear un dialogo permanente entre los padres y los maestros. Este dialogo puede funcionar dentro de una estructura operada por la institución educativa o implementada por una institución mayor, por ejemplo, una que represente al Estado y que influya en la agenda docente. En cualquier caso, para que la participación de los padres opere, estos deben tener una influencia real sobre las decisiones de la institución educativa, de forma que haya un vínculo directo entre sus opiniones y las estrategias ejecutadas efectivamente dentro de la institución. Para que esto suceda es imperativo que los maestros cedan cierto margen de dirección en la toma de decisiones pedagógicas y educativas, ya que mantener una postura de poder que impida que la participación de los padres tenga algún tipo de implicación, lo que produce es una reducción paulatina de la participación de los padres y a la postre una reducción en la calidad académica, por lo que se abre una paradoja debido a que los maestros consideran que la intervención de los padres es innecesaria ya que son los maestros los expertos pedagógicos y sin embargo, su participación aislada, sin la construcción conjunta con los padres, reduce la calidad educativa (Bertrand \& Deslandes, 2005; Coninck, Dotger, Vanderlinde, R. \& Walker, 2020). 
Sin embargo, esta paradoja surgida debido a que los maestros consideran que la particiáción de los padres puede reducir la calidad educativa y en consecuencia, no integran en la toma de decisiones a los padres y por lo tanto, terminan reduciendo la calidad educativa; tiene fundamento en que los maestros parecen no estar preparados para incorporar a la familia activamente e incluso, en ocasiones muestran resistencia a esto. Las razones de esto son múltiples y cobijan desde la autopercepción del maestro, hasta la falta de las habilidades necesarias para la participación en comunidad. En este sentido, las razones psicologías cobijan la autopercepción de fracaso, en cuyo caso, los maestros no se sienten con las habilidades para integrar a otros actores debido en parte a que no están dispuestos a recibir una revisión detallada de su trabajo ni de la calidad de este, ya que ajeno o no a la realidad, la sola idea de poder ser vigilados dentro del ambiente educativo es intolerable. Así mimos, hay un vacío dentro de su formación, esto debido a que los hallazgos sobre la participación de los padres, si bien datan de hace décadas, es una práctica que aún no ha sido suficientemente extendida dentro de las facultades en las que se forman los maestros y por lo tanto no se conoce en detalle del impacto positivo que acarrea la participación de los padres (Martijn, Mutton, Thompson \& Vanderlinde, 2018; Guo \& Kilderry, 2018; Eva \& Mei, 2019; Hoover, Jones, Reed \& Walker, 2002)

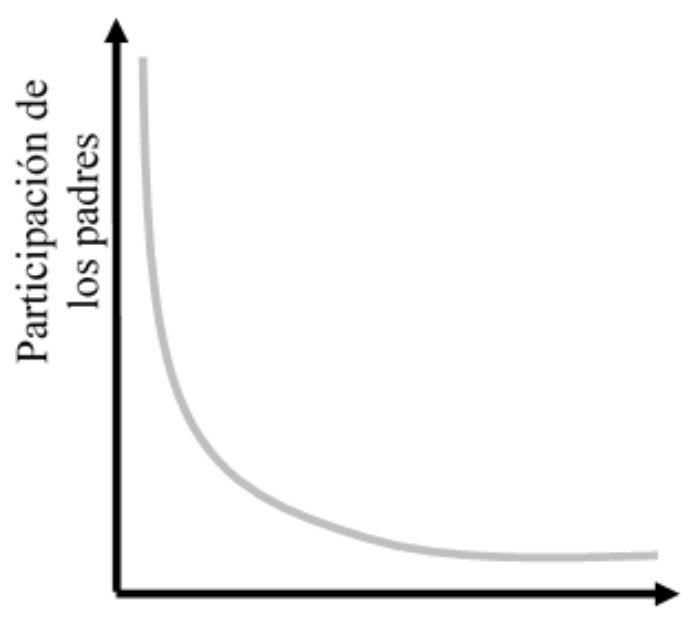

Barreras culturales e imaginarios negativos de los maestros

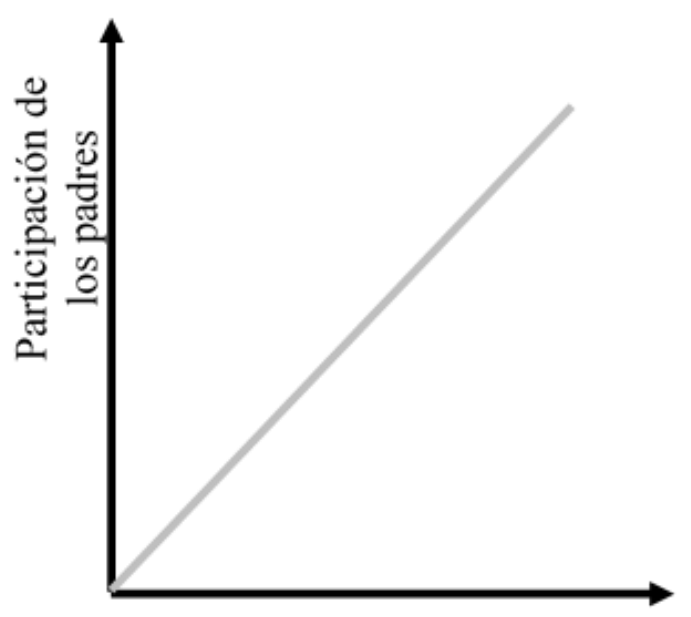

Creencias positivas de los frente a la participación de los padres

Figura 1. Creencias de los maestros.

Fuente: Elaboración del autor basado en las investigaciones de Martijn et al. (2018), Hoover et al. (2002), Eva y Mei (2019), Guo y Kilderry (2018), Aguirre et al. (2013) y Bakker et al. (2007).

Hay una razón más poderosa que influye en la forma en que se relacionan los maestros con la idea de la participación de los padres, y son los imaginarios que cada docente posee. En este sentido, los imaginarios de cada docente sobre el tipo de cultura buena y el tipo de sociedad mala, la concepción de lo que es logro académico e incluso la concepción de lo que podría ser éxito profesional de cada uno de los maestros puede influir directamente en la forma en la que los maestros se relacionan con los padres. Si dentro de la cosmovisión de un maestro las personas de origen indígena son inferiores (negando la riqueza cultural, filosófica, intelectual, las practicas autosostenibles y la vida en comunidad) tendrá impulsos inconscientes que lleven a menospreciar 
la participación de los padres de un niño de origen indígena. Si por el contrario su idea de un escenario pedagógico está más relacionada con los bienes materiales, tendrá una mayor motivación para facilitar la participación de los padres que mayor capital económico posean, en detrimento incluso de los otros padres. Estas barreras no son solamente negativas, sólo que son estas las que interfieren en la relación maestros-padres (Aguirre et al., 2013; Bakker, Brus \& Denessen, 2007).

\section{CONCLUSIÓN}

Los maestros son actores clave en el fomento de la participación de los padres, por lo que, si estos creen que la participación de los padres es deseable, pueden crear estrategias para que estos efectivamente participen. Aunque si los maestros tienen barreras culturales o imaginaros negativos frente a la participación de los padres, estos provocarán una menor participación de los padres. Luego, si los maestros creen que los padres (todos en general) pueden afectar positivamente en la educación de sus hijos y, actúan, estos efectivamente lo harán.

El papel de las creencias de los maestros en la participación familiar se compone de varias aristas que integran comunidad, comunicación, formación e imaginarios. Se reconoce que la relación maestro-padres afecta positivamente el aprendizaje de los niños, por lo que favorecer la comunicación entre ellos permite que en comunidad se logre el objetivo. No obstante, barreras como la formación docente y los imaginarios que estos mismos poseen, reducen la posibilidad de esta integración.

Lo interesante de los hallazgos es que se evidencia que esto sucede en diferentes culturas y países, dado que la influencia de nuestras creencias es algo que concierne al ser humano. Trabajar en reconocer dichas barreras e imaginarios puede ser importante para hacer la transformación cultural, aunque desde un inicio se debe reconocer que puede ser un proceso lento (al tratarse de algo tan arraigado como nuestros imaginarios), lo cual implica que entre más rápido se inicie la transformación, mejor.

Agradecimientos / Acknowledgments:

Expreso mi agradecimiento a los docentes del Programa del doctorado en educación de la Unidad de posgrado de la Universidad Autónoma de Tlaxcala, México.

Conflicto de intereses / Competing interests:

El autor declara que no incurre en conflictos de intereses.

Rol de los autores / Authors Roles:

No aplica.

\section{Fuentes de financiamiento / Funding:}

El autor declara que no recibió un fondo específico para esta investigación.

\section{Aspectos éticos / legales; Ethics / legals:}

El autor declara no haber incurrido en aspectos antiéticos, ni haber omitido aspectos legales en la realización de la investigación. 


\section{REFERENCIAS BIBLIOGRÁFICAS}

Aguirre, J., Drake, C., Foote, M., Gau, T., Roth, A., \& Turner, E. (2013). Orientations of prospective teachers toward students' family and community. Teaching and Teacher Education, 35, 126-136. doi:10.1016/j.tate.2013.06.003

Ali, A., Alizadeh, M., \& Tehranian, A. (2018). A Study on the Changes on Teachers' Knowledge and Beliefs After a Workshop Based on Mathematics Education Software, by Relying on Fuzzy Method. PNA, 13(1), 19-40. doi:10.30827/pna.v13i1.6593

Amaya, E., Delgado, A., Gnecco, L., León, E., \& Valencia, D. (2014). Creencias de un grupo de trece profesores sobre las condiciones laborales que dignifican y precarizan su profesión. Universidad San Buenaventura, $156 . \quad$ Retrieved from http://biblioteca.usbbog.edu.co:8080/Biblioteca/BDigital/83580.pdf

Anzaldo, G., \& Benavides, G. (2020). Expectativas en las tasas de interés y noticias de política monetaria de EEUU. Revista Mexicana de Economía y Finanzas, 17(1), 17-35. doi:10.21919/remef.v15i1.463

Anzoátegui, J., \& Galvis, J. (2019). Efectos de los anuncios de política monetaria y la credibilidad sobre las expectativas de inflación: evidencia para Colombia. Apuntes del Cenes, 38(67), 73-94. doi:10.19053/01203053.v38.n67.2019.7912

Arreola, C., Carlos, E., \& Valdés, Á. (2013). Desarrollo de un instrumento para medir la participación de los padres en la educación escolar de los hijos. Revista de evaluación educativa, 2(1), 92-109. $\quad$ Retrieved from https://www.researchgate.net/publication/285871818_Desarrollo_de_un_instrumento_par a_medir_la_participacion_de_los_padres_en_la_educacion_de_los_hijos

Astorquiza, B. (2018). Efectos de la pedagogía y el compromiso educativo sobre el logro académico de los estudiantes de educación media. Revista de Métodos Cuantitativos para la Economía y la Empresa, 28, 43-67. Retrieved from https://upo.es/revistas/index.php/RevMetCuant/article/view/2765/3842

Bakker, J., Brus, M., \& Denessen, E. (2007). Socio-economic background, parental involvement and teacher perceptions of these in relation to pupil achievement. Educational Studies, 33(2), 177-192. doi:10.1080/03055690601068345

Bayas, C., Molina, L., Ordóñez, E., \& Rodríguez, A. (2018). Creencias y prácticas pedagógicas alrededor del clima en el aula frente al problema de las drogas. Revista Lasallista de Investigación, 15(2), 141-152. doi:10.22507/rli.v15n2a11

Bazán, A., Hernández, E., Hernández, C., \& Ochoa, E. (2020). Relaciones entre contexto de procedencia y logro académico al terminar la primaria. Revista Electrónica de Investigación Educativa, 22(e08), 1-16. doi:10.24320/redie.2020.22.e08.2335 
Becker, D., \& Wessling, K. (2020). The impact of classroom, school, neighborhood, and institutional factors on teachers' expectations. Maastricht, Holanda: Research Centre for Education and the Labour Market. doi:10.26481/umaror.2020004

Bendixsen, S., \& Danielsen, H. (2020). Great expectations: migrant parents and parent-school cooperation in Norway. Bergen, Norway: Comparative Education. doi:10.1080/03050068.2020.1724486

Bertrand, R., \& Deslandes, R. (2005). Motivation of Parent Involvement in Secondary-Level Schooling. The Journal of Educational Research, 98(3), 164-175. doi:10.3200/JOER.98.3.164-175

Boonk, L., Gijselaersa, J., Ritzen, H., \& Saskia, B. (2018). A review of the relationship between parental involvement indicators and academic achievement. Educational Research Review(24), 10-30. doi:10.1016/j.edurev.2018.02.001

Bronfenbrenner, U. (1979). The Ecology of Human Development. Cambridge: Harvard University Press. $\quad$ Retrieved from https://khoerulanwarbk.files.wordpress.com/2015/08/urie_bronfenbrenner_the_ecology_ of_human_developbokos-z1.pdf

Bronfenbrenner, U., \& Morris, P. (2006). handbook of child psychology. volume one: theoretical models of human development. In W. Damon, R. Lerner, \& R. Lerner (Ed.), Handbook of child psychology. Volume 1: theoretical models of human development (Sexta ed., pp. 793828). New Jersey: Wiley. Retrieved from http://edfa2402resources.yolasite.com/resources/BronfenbrennerModelofDevelopment.pd $\mathrm{f}$

Bronfenbrenner, U., \& Morris, P. (2016). The Bioecological Model of Human Development. In R. Lerner, W. Damo, \& R. Lerner (Eds.), Handbook of Child Psicoligy. Volume 1: Teorical Models of Human Developmen (Sexta ed., pp. 793-828). Hoboken: Jhon, Wiley and Sons, Inc. $\quad$ Retrieved from http://edfa2402resources.yolasite.com/resources/BronfenbrennerModelofDevelopment.pd $\mathrm{f}$

Carlos, E., Urías, M., Valdéz, Á., \& Wendlandt, T. (2016). Desarrollo de una Escala para Medir Prácticas Docentes para Involucrar a las Familias en la Educación en México. Archivos Analíticos de Políticas Educativas, 24(101), 1-13. doi:10.14507/epaa.v24.2452

Coninck, K., Dotger, B., Vanderlinde, R., \& Walker, J. (2020). Measuring student teachers' selfefficacy beliefs about family-teacher communication: Scale construction and validation. $\begin{array}{llll}\text { Studies in Educational } & \text { Evaluation, } & \text { 64, }\end{array}$ doi:https://doi.org/10.1016/j.stueduc.2019.100820

Corral, V., Frías, M., González, D., \& Miranda, J. (1998). Relaciones entre variables de apoyo familiar, esfuerzo académico y rendimiento escolar en estudiantes de secundaria: un modelo estructural. Enseñanza e Investigación en Psicología, 3(1), 157-167. 
Dallaire, J., Hoover, K., Sandler, H., Walker, J., \& Wilkins, A. (2005). Parental Involvement: Model Revision through Scale Development. The Elementary School Journal, 106(2), 85104. Retrieved from http://www.jstor.org/stable/10.1086/499193

Danna, J., Londoño, D., \& Stellian, R. (2020). Expectativas semiadaptativas en los modelos macroeconómicos multiagentes. Una aplicación al análisis de la fragilidad financiera empresarial. Revista de Economía del Rosario, 23(1), 65-108. doi:10.12804/revistas.urosario.edu.co/economia/a.8627

Daza, G., \& Garza, B. (2020). Estudio de las expectativas de estudiantes mexicanos del nivel medio superior con respecto al Cálculo Diferencial e Integral. Educação Matemática Pesquisa, 22(1), 610-631. doi:10.23925/1983-3156.2020v22i1p610-631

Derin, D., Noftle, J., Quintanar, A., \& Warren, S. (2011). Preparing Urban Teachers to Partner with Families and Communities. The School Community Journal, 21(1), 91-112. Retrieved from https://files.eric.ed.gov/fulltext/EJ932202.pdf

Duckworth, A., Eskreis, L., Galla, B., Gross, J., \& Taxer, J. (2019). Self-Control and Academic Achievement. Annual Review of Psychology, 70, 373-399. doi:10.1146/annurev-psych010418-103230

Epstein, J. (1986). Parents' Reactions to Teacher Practices of Parent Involvement. The Elementary School Journal, 86(3), 277-294. Retrieved from http://www.jstor.org/stable/1001545

Eva, H., \& Mei, N. (2019). Are they ready for home-school partnership? Perspectives of kindergarten principals, teachers and parents. Children and Youth Services Review, 99, 1017. doi:10.1016/j.childyouth.2019.01.019

Fuentes, V., Guzmán, C., Quevedo, K., \& Villablanca, I. (2013). Creencias docentes de profesores ejemplares y su incidencia en las prácticas pedagógicas. Estudios Pedagógicos, 39(2), $97-$ 113. Retrieved from https://scielo.conicyt.cl/pdf/estped/v39n2/art07.pdf

Green, C., Hoover, K., Sandler, H., \& Walker, J. (2007). Parents' motivations for involvement in children's education: An empirical test of a theoretical model of parental involvement. Journal of Educational Psychology, 99(3), 532-544. doi:10.1037/0022-0663.99.3.532

Guo, K., \& Kilderry, A. (2018). Teacher accounts of parent involvement in children's education in China. Teaching and Teacher Education, 69, 95-103. doi:10.1016/j.tate.2017.09.018

Hoover, K., \& Sandler, H. (1997). Why do parents become involved in their children's education? Review of Educational Resear, 67(1), 3-42. Retrieved from https://journals.sagepub.com/doi/10.3102/00346543067001003

Hoover, K., Jones, K., Reed, R., \& Walker, J. (2000). Parents' Motivations for Involvement in Children's Education: Testing a Theoretical Model. Annual Conference of the American Educational Research Association, (pp. 1-21). New Orleans. 
Hoover, K., Jones, K., Reed, R., \& Walker, J. (2002). Teachers Involving Parents (TIP): results of an in-service teacher education program for enhancing parental involvement. Teaching and Teacher Education, 18(7), 1-21. Retrieved from http://meparentsaspartners.pbworks.com/f/TIP_paper.pdf

Hornby, G., \& Lafaele, R. (2011). Barriers to parental involvement in education: An explanatory model. Educational Review, 63(1), 37-52. doi:10.1080/00131911.2010.488049

Juang, L., Noack, P., Schachner, M., Vietze, J., \& Vijver, F. (2020). Juggling between parental and school expectations: the development ofdomain-specific acculturation orientations in early adolescence. Journal of Research on Adolescence, 1-17. doi:10.1111/jora.12547

Keyes, C. (2000). Parent-Teacher Partnerships: A Theoretical Approach for Teachers. Actas del Simposio Lilian Katz, (pp. 107-118). Champaign. Retrieved from https://eric.ed.gov/?id=ED470883

Luna, D., \& Velázquez, P. (2019). Evaluación del impacto del Programa de Escuelas de Tiempo Completo en medidas de logro académico de centros escolares en México. Revista Latinoamericana de Estudios Educativos, 49(2), 87-120. Retrieved from https://www.redalyc.org/jatsRepo/270/27059273013/27059273013.pdf

Martijn, T., Mutton, T., Thompson, I., \& Vanderlinde, R. (2018). Family-school partnerships: a challenge for teacher education. Journal of Education for Teaching, 44(3), 252-257. doi:10.1080/02607476.2018.1465545

Meenu, D. (2016). Factors Affecting the Academic Achievement: A Study of Elementary School Students of NCR Delhi, India. Journal of Education and Practice, 7(4), 70-74. Retrieved from https://files.eric.ed.gov/fulltext/EJ1092343.pdf

Ramírez, A., Velasco, F., \& Vera, J. (2015). Procesos escolares y eficiencia interna: ¿cuál es la relación con el logro académico en la educación media superior mexicana? Archivos Analíticos de Políticas Educativas, 23(53), 1-21. doi:10.14507/epaa.v23.1735

Torrico, E., Santín, C., Villas, M., Menéndez, S., \& López, M. (2002). El modelo ecológico de Bronfrenbrenner como marco teórico de la psicooncología. Anales de Psicología, 18(1), 45-59. Retrieved from https://www.um.es/analesps/v18/v18_1/03-18_1.pdf 